\title{
THE PROPOSED RADIO SCHMIDT TELESCOPE: THE TECHNICAL CHALLENGES
}

\author{
P.E. DEWDNEY and T.L. LANDECKER \\ Dominion Radio Astrophysical Observatory, Penticton, B.C., V2A 6K3
}

\begin{abstract}
The concept of the Radio Schmidt Telescope is designed to fill a scientific requirement for a radio telescope which is very sensitive to diffuse, extended emission, but has much higher resolution than singleantenna telescopes. This telescope undertakes to utilize a new region of "parameter space" in the aperture synthesis technique. The emphasis of this article is on a process leading to the best implementation of the concept at reasonable cost while satisfying all the scientific objectives.
\end{abstract}

There is a scientific need for a telescope which extends the capability of the world's largest single-antenna radio telescopes to higher resolution without significantly sacrificing sensitivity to diffuse emission. Studies of extended HI emission, low-brightness continuum emission, distributed spectral-line emission, solar studies and other problems all require sensitivity to extended emission and/or rapid imaging capability and would benefit crucially from an increase in angular resolution. The aperture synthesis technique remains the only method available to us to deliver the required combination of resolution and sensitivity at $\mathrm{cm}$ and $\mathrm{m}$ wavelengths. The Very Large Array (VLA) in its $\mathrm{D}$ configuration is the current best approximation. Nevertheless, there is a gap between what is possible with the VLA, and what is required.

The salient characteristics of a telescope which is well suited for such applications are: a wavelength range from about $1 \mathrm{~m}$ to $1 \mathrm{~cm}$; a large collecting area arranged in an array configuration which is optimum for the scientific requirements (a 2-dimensional array configuration which is fairly compact in wavelengths); a comparatively wide (instantaneous) field-of-view; sufficient speed for high fidelity imaging of large areas of sky containing complex structure (areas much larger than the instantaneous field-of-view); spectral line capability sufficient to handle all known lines in the $\mathrm{cm}-$ to-m range of wavelengths.

The above characteristics imply both a large collecting area and a large field-of-view, and these are competing requirements. The difficulty can be solved in principle by using many small antennas. (Note that "small" is a relative term, and that actual optimization of parameters may yield antenna diameters of many meters). Building a telescope with many small antennas presents considerable instrumental problems but also confers some important advantages. The number of baselines between antennas increases as the square of the number of antennas, thus requiring a very large correlator. But many 
baselines also confer high dynamic range, high speed, and good sky coverage. The primary goal of a detailed design of such a telescope would be to achieve the best balance in these basic parameters.

We consider, as a starting point, a telescope with the following parameters: an array of 100 antennas with diameter $12 \mathrm{~m}$ each with frequency coverage in four bands, namely $22,5,1.6 / 1.4$ and $0.4 \mathrm{GHz}$. (Total physical collecting area is about $11300 \mathrm{~m}^{2}$ ). The precise array configuration(s) is (are) to be established, but there could be two configurations, a compact one with an east-west dimension of several 100's of meters, and a large one about $2 \mathrm{~km}$ across. In any case these dimensions represent the limiting configuration sizes which would be contemplated.

In the larger configuration this telescope would be able to map a $1.5^{\circ}$ field-of-view at a wavelength of $21 \mathrm{~cm}$ with a continuum sensitivity (total intensity in a $50 \mathrm{MHz}$ band) to extended structure of $57 \mathrm{mK}$ in 1 hour $(16 \mathrm{mK}$ in 12 hours) at the field center, and would have sensitivity to unresolved sources of $26 \mu \mathrm{Jy}$ in 1 hour ( $7.5 \mu \mathrm{Jy}$ in 12 hours). (It is noted that the confusion limit will be reached at the lowest frequency.) The resolution would be about $1.3^{\prime \prime}, 6^{\prime \prime}, 20^{\prime \prime}$ and $70^{\prime \prime}$ at the $22,5,1.5$, and $0.4 \mathrm{GHz}$ frequencies, respectively. In a typical spectrometer bandwidth of $10 \mathrm{kHz}$ the sensitivity would be about $4.0 \mathrm{~K}$ in 1 hour ( $1.1 \mathrm{~K}$ in 12 hours) at full spatial resolution at the field center using power from both polarizations.

In the smaller configuration the sensitivity to extended structure would increase by a factor of about 100 , in proportion to the area of the synthesized beam. This is particularly important for observations of weak spectral lines, especially at high frequencies. In a $10 \mathrm{kHz}$ (spectrometer) bandwidth the sensitivity would be about $40 \mathrm{mK}$ in one hour $(10 \mathrm{mK}$ in 12 hours $)$. The resolution would be about $13^{\prime \prime}, 60^{\prime \prime}, 3^{\prime}$ and $12^{\prime}$ at the $22,5,1.5$, and $0.4 \mathrm{GHz}$ frequencies, respectively.

The telescope's potential for high dynamic range would ensure that these limits could be met even in the presence of relatively bright sources. For bright objects, such as the Sun or Galactic HI-emission, the telescope would have camera-like imaging qualities. However, for observations of very weak emission (e.g. the $3 \mathrm{~K}$ background absorbed by molecular lines), long integrations would be required. With its large collecting area the telescope would also be a powerful node for both ground and space based VLBI, and for other types of observation where raw collecting area is important.

Is such a telescope design achievable? A synthesis instrument with this many baselines $(\sim 5000)$, requiring a very large digital correlator, has never been built, particularly for spectral-line work. There is no doubt that technology exists to build the telescope, and technical developments have reached the stage where it probably can also be built at an affordable cost. We are relying on improvements in technology (over the state of technology used in current telescopes) in three key areas: inexpensive (HEMT) low-noise receivers are available now that were not available to the designers of, for example, the VLA; communications technology utilizing inexpensive digital electronics and fiber-optic transmission lines now allows transmission of unprecedented information-bandwidths from numerous locations (this technology is already in use in the Australia Telescope); integrated-circuit technology continues to show almost exponential growth in performance-to-cost ratio. New design 
tools are available which will also reduce the development costs of very complex systems.

\section{BALANCING COST AND PERFORMANCE}

In this section we outline a process by which further work in designing the Radio Schmidt Telescope can proceed, followed by a few, more detailed examples.

The above set of design parameters for the telescope is meant to be used as a starting point for a (possibly iterative) process of optimization. This process is much too complex to be described in rigorous mathematical terms. Nevertheless, the goal is the same, to maximize the ratio of performance to cost, under a number of constraints. In this case the total cost will have to be constrained. (Otherwise both cost and performance will increase without bound). The current overall cost target is about $45 \times 10^{6} \$ \mathrm{CDN}$. The basic concept of the telescope and its related scientific goals must also be translated into constraints. A sensible definition of performance can be made from a (weighted) combination of carefully chosen factors. Ideally, a performance factor is related to some scientifically important feature of the telescope and happens to be closely tied to an isolated cost item.

Given the scientific objectives, the dominant performance factors for the Radio Schmidt Telescope are: total effective area, size and flexibility of the array configuration, telescope speed (dependent on instantaneous fieldof-view and instantaneous $u-v$ coverage), bandwidth, frequency coverage, channelization, and image quality (dependent on system noise, $u-v$ coverage and errors which affect mosaicing). Most of these are self-evident choices. Ultimately, scientific requirements will dictate how much weight each of these factors is given.

Cost is easier to define than performance, but there are complex tradeoffs between the original capital cost, cost of improvements, and operating cost. The individual contributions to the cost of the telescope can be identified almost by following the signal path through the system. In the case of the capital cost, seven major sub-systems dominate: antennas (and feeds), antenna-based electronics, array configuration costs (includes site servicing, civil works and some data communication costs), correlator, imaging system, and infrastructure (includes buildings, etc.).

As a first approximation to overall optimization, the performance-tocost ratio of each of these sub-systems can be maximized separately. It should be noted that some of the cost contributions are strong functions of time (e.g. digital electronics), and therefore, some prediction of the behaviour of the performance-to-cost ratio will be necessary. In almost every instance, however, the trend is beneficial (i.e. the performance-to-cost ratio increases with time).

The case of antennas is exemplary. They are probably the most important sub-system, and represent about $30 \%$ of the total capital cost. Furthermore, their cost is expected to be stable (except for inflation). A recent study of the design of antennas in the $12 \mathrm{~m}$ class indicates that, for a cost increase of about $20 \%$, the operating frequency can be raised from $5 \mathrm{GHz}$ to $25 \mathrm{GHz}$. The extra cost arises mainly from the need for more accurate reflector panels. In this range of diameters, the structure will have adequate stiffness, and, if included 
in the original design, the extra cost of sufficiently accurate machined parts (bearings, etc.) is small. It is clear, however, that high frequency capability must be planned for from the start. The study also indicated that it is feasible to build transportable antennas in this range of sizes. The ability to make easily transportable antennas allows considerable freedom to consider more than one array configuration.

The configuration of the array is another facet of the design requiring a careful balance of cost and performance. It appears that two configurations will be necessary to meet the full range of scientific objectives. (The sizes given above represent the extremes.) The sensitivity to extended emission is dependent directly on the area of the synthesized beam. Since there is a factor of about 70 in the range of observing wavelengths, the beam area becomes too small in large configurations to reach acceptable brightnesstemperature sensitivity for interesting astrophysics in the $22 \mathrm{GHz}$ band. The impact on the cost of a re-configurable array is mainly in the provision of more serviced antenna-stations, and in the operational cost of reconfiguration (assuming, as noted above, that transportable antennas cost about the same as fixed antennas). An intensive study of array designs for the telescope may reveal a nearly optimum configuration which serves the astrophysics adequately, and does not "waste" very much collecting area in any particular type of observation. Adding a new configuration also falls in the category of improvements which can be added later. It seems intuitively clear that such a configuration would have to be very centrally condensed in order to serve the astrophysical applications at high frequencies.

The correlator is mentioned above as a potential feasibility (or cost) barrier. At the time (1985) that this project was first discussed with colleagues, this was the most frequently mentioned concern. Since then, a fairly general analysis of the cost of correlators has been done at the National Radio Astronomy Observatory. When applied to this case, the analysis yields a current cost of about $4.3 \times 10^{6} \$ \mathrm{U}$.S. plus an estimated design cost of about $1.3 \times$ $10^{6}$ \$U.S. (Total $=6.7 \times 10^{6} \$ \mathrm{CDN}$, about $15 \%$ of the target for the total capital cost). In about five years costs for suitable digital circuits are expected to have dropped by a factor of about 10 . The cost of correlators of this size scales approximately as the product of the number of antennas and the bandwidth. It is now questionable whether the "starting point" design is well matched to the project because the correlator is too small. However, increasing the overall bandwidth of the correlator may not yield much astrophysical return for the target range of beamsizes. The real astrophysical need lies in more sensitivity to weak spectral lines. In general terms this can only be provided by more collecting area or less system noise. It appears, therefore, that the parameters for the correlator cannot be optimized separately, but must intimately take part in more global optimization.

As pointed out above, the development of low cost (HEMT) amplifiers has allowed the contemplation of telescope designs like the Radio Schmidt. However, a substantial gain in sensitivity could be achieved by cooling the amplifiers. At $\mathrm{T}_{\text {phy }}=300 \mathrm{~K}, \mathrm{~T}_{\text {rec }} \approx 15 \mathrm{f}_{\mathrm{GHz}}$; at $\mathrm{T}_{\text {phy }}=20 \mathrm{~K}, \mathrm{~T}_{\text {rec }} \approx 2.5 \mathrm{f}_{\mathrm{GHz}}$. There is an approximately linear improvement in receiver noise as physical temperature falls. The capital cost of cryogenic cooling of receivers is not impossibly high, but the maintenance cost (in personnel) is unacceptably high. 
More suitable methods of cooling are being investigated, which will probably have the ability to cool the amplifiers to temperatures $\approx 80 \mathrm{~K}$.

In conclusion we have described the concept of the Radio Schmidt

Telescope, outlined a particular implementation of the concept, described the process of optimization that any such design must undergo, and discussed some important examples where refinement of the design can take place.

Richard Simon: How did you arrive at the Strawman size of $12 \mathrm{~m}$ ? Was this driven by cost, field of view, or what?

Peter Dewdney: The initial collecting area was determined as being at least that of the VLA. The strawman design was initially based on the feeling that correlators would be very expensive with more than 5000 baselines (100 antennas). This, however, needs to be further optimized. 